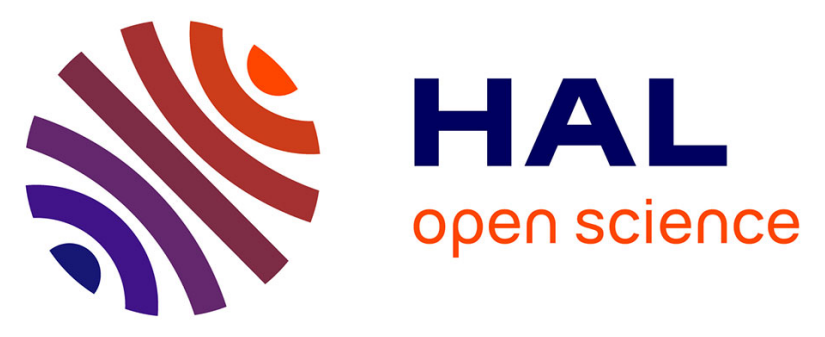

\title{
Tuning the work function of monolayer graphene on 4H-SiC (0001) with nitric acid
}

Fethullah Günes, Hakim Arezki, Debora Pierucci, David Alamarguy, José

Alvarez, Jean-Paul Kleider, Yannick J. Dappe, Abdelkarim Ouerghi,

Mohamed Boutchich

\section{To cite this version:}

Fethullah Günes, Hakim Arezki, Debora Pierucci, David Alamarguy, José Alvarez, et al.. Tuning the work function of monolayer graphene on $4 \mathrm{H}-\mathrm{SiC}$ (0001) with nitric acid. Nanotechnology, 2015, 26 (44), pp.445702. 10.1088/0957-4484/26/44/445702 . hal-01244484

HAL Id: hal-01244484

https://hal-centralesupelec.archives-ouvertes.fr/hal-01244484

Submitted on 4 Dec 2020

HAL is a multi-disciplinary open access archive for the deposit and dissemination of scientific research documents, whether they are published or not. The documents may come from teaching and research institutions in France or abroad, or from public or private research centers.
L'archive ouverte pluridisciplinaire HAL, est destinée au dépôt et à la diffusion de documents scientifiques de niveau recherche, publiés ou non, émanant des établissements d'enseignement et de recherche français ou étrangers, des laboratoires publics ou privés. 


\title{
TUNING THE WORK FUNCTION OF MONOLAYER GRAPHENE ON 4H-SIC (0001) WITH NITRIC ACID
}

Fethullah Günes ${ }^{1, \text { a }}$, Hakim Arezki ${ }^{1}$, Debora Pierucci ${ }^{2}$, David Alamarguy ${ }^{1}$, José Alvarez ${ }^{1}$, Jean-Paul Kleider ${ }^{1}$, Yannick J. Dappe ${ }^{3}$, Abdelkarim Ouerghi ${ }^{2}$ and Mohamed Boutchich ${ }^{1}$

${ }^{1}$ GeePs, CNRS UMR8507, CentraleSupelec, Univ Paris-Sud, Sorbonne Universités-UPMC Univ Paris 06, 11 rue Joliot-Curie, Plateau de Moulon, 91192 Gif-sur-Yvette Cedex, France

${ }^{2}$ Laboratoire de Photonique et de Nanostructures (CNRS - LPN), Route de Nozay, 91460 Marcoussis, France

${ }^{3}$ Service de Physique de 1'Etat Condensé (UMR 3680 CNRS), IRAMIS, CEA Saclay, 91191, Gif-Sur-Yvette, France

\begin{abstract}
Chemical doping of graphene is a key process for the modulation of its electronic properties and the design and fabrication of graphene based nanoelectronic devices. Here, we study the adsorption of diluted concentrations of nitric acid $\left(\mathrm{HNO}_{3}\right)$ onto monolayer graphene/4H-SiC (0001) to induce a variation of the graphene work function (WF). Raman spectroscopy indicates an increase in the defect density subsequent to the doping. Moreover, ultraviolet photoemission spectroscopy (UPS) was utilized to quantify the WF shift. UPS data show that the WF of the graphene layer decreased from $4.3 \mathrm{eV}$ (pristine) down to $3.8 \mathrm{eV}\left(30 \% \mathrm{HNO}_{3}\right)$ and then increased to 4.4 $\mathrm{eV}$ at $100 \% \mathrm{HNO}_{3}$ concentration. These observations were confirmed using density functional theory (DFT) calculations. This straightforward process allows a large WF modulation rendering the molecularly modified graphene/4H-SiC(0001) a highly suitable electron or hole injection electrode.
\end{abstract}

KEYWORDS: Graphene, Work function, doping, Raman spectroscopy, UPS spectroscopy, DFT calculations.

*mohamed.boutchich@geeps.centralesupelec.fr

a) Department of Materials Science and Engineering,

Izmir Kâtip Çelebi University Cigli Main Campus

35620, IZMIR, Turkey 


\section{Introduction}

Graphene is a 2D-material combining various outstanding properties in a single material, like quantum Hall effect or massless Fermions among others ${ }^{1,2}$. Its high mobility, optical transparency, along with flexibility, high mechanical strength and environmental stability make it a very attractive material for a broad range of applications $^{3-4}$. Graphene can also be exploited for precise metrological applications, as it has been demonstrated recently with exceptionally accurate measurements of the quantum Hall resistance quantization 5,6 . In order to implement the potential of such material into viable applications, large-scale wafers of high quality graphene grown on insulating substrates are required for device integration ${ }^{7,8}$. Moreover, to achieve performant devices, the electronic properties of the material must be modulated ${ }^{9,10,11}$. In that respect, the work function (WF) is a remarkable property that can be tuned for various optoelectronic applications like transparent electrodes for optoelectronic devices, or multiple tunnel heterostructures matching the energy offsets of common transport materials, to achieve low and/or large charge injection barriers ${ }^{12}$. To reach this goal, the modification of the work function of graphene has to be obtained without damaging the overall characteristics of the material. A possible approach to vary the WF is to modify the surface by depositing a monolayer of a molecular donor or acceptor. Recent studies have demonstrated possible WF variations using as acceptor materials tetrafluoro-tetracyanoquinodimethane (F4-TCNQ), gold colloids or metals ${ }^{13,14,15}$ for example. Although these methods have demonstrated WF modulation, they present major drawbacks. On one hand, the transparency of graphene is reduced, which represents a strong limitation for optoelectronic applications where graphene could play a role. On the other hand, these adsorbed elements create scattering interfaces that may severely affect the transport properties of graphene. At last, deposition and temperature dependence are potential limitations for the device fabrication process. Among the dominant graphene synthesis methods, chemical vapor deposition (CVD) and epitaxy on SiC substrate are the leading technologies in terms of scale and properties. For electronic applications, the growth of graphene by CVD on metals requires transferring the graphene onto an insulating substrate. This last operation involves chemicals such as PMMA and copper etchant known to induce a $p$-type doping of the material ${ }^{16}$. Although this approach allows the transfer of graphene on any functional substrate, the electronic properties of the graphene sheet are altered by these manipulations. Furthermore, epitaxial graphene on hexagonal silicon carbide ( $\mathrm{SiC}$ ) wafers presents homogeneous films with large areas and high electron mobility ${ }^{17}$. The $\mathrm{SiC}$ substrate is suitable for opto-electronic applications since it is transparent over a very broad frequency spectrum. It has a large thermal conductivity, and is compatible with highfrequency devices where losses due to residual conductivity of the substrate have to be minimized by using insulating materials. We have recently demonstrated the WF modulation on trilayer graphene on $\mathrm{SiC}(0001)$ using in-situ nitrogen flux ${ }^{18}$. Furthermore, Das et al have demonstrated that Nitric Acid $\left(\mathrm{HNO}_{3} / \mathrm{NA}\right)$ on CVD graphene is an efficient doping agent inducing $p$-type variation of the $\mathrm{WF}^{19,20}$.

In this work, we have monitored the structural and electronic modifications of epitaxial graphene for different concentrations of diluted Nitric Acid (NA) 15 vol\% (NA15), 30 vol\% (NA30), 70 vol\% (NA70) and $100 \%$ (NA100) of nitric acid (NA) solutions in deionized water (DI water) for $2 \mathrm{~min}$. The electronic properties of the doped graphene samples were characterized by Raman spectroscopy. The changes in work function after NA doping were measured using ultraviolet photoemission spectroscopy (UPS). Based on these observations, the influence of NA doping on graphene work function is discussed. To highlight the effect of nitric acid doping, these measurements were compared with the obtained values for pristine graphene. We demonstrate in the 
present work that the NA doping permits the WF modulation with respect to the dilution. Indeed, the WF first increases the electronic doping between 15 and 30 vol\% (NA15, NA30) and then decreases the electronic doping between 70 and 100 vol\% (NA70, NA100). This study on epitaxial graphene presents striking differences compared to the work of Das et al on CVD graphene where only $p$-type work function shifts is observed. We clearly demonstrate that both systems react differently to an identical chemical functionalization. These observations are supported by density functional theory calculations (DFT).

\section{Experimental section}

The graphene was grown on semi-insulating $\mathrm{SiC}(0001)$ substrates ${ }^{17,21}$. The $\mathrm{SiC}$ sample was then heated to $1000{ }^{\circ} \mathrm{C}$ in a UHV chamber and further heated to $1525^{\circ} \mathrm{C}$ in an $\mathrm{Ar}$ atmosphere to favor the formation of large and homogeneous graphene layer. The substrate was then cooled down from $1525^{\circ} \mathrm{C}$ to room temperature $^{18,22}$. The samples were subsequently transferred ex situ from the growth chamber to the AFM microscopy, Raman spectroscopy and UPS measurement systems.

The morphology of the nitrogen doped epitaxial graphene was studied after growth by atomic force microscopy (AFM). The Raman measurements were carried out with a frequency-doubled NdYag laser operating at $532 \mathrm{~nm}$ as excitation source with up to $20 \mathrm{~mW}$ continuous wave output power. The spectra were recorded through a 300 $\mathrm{mm}$ imaging spectrometer equipped with both a 600 lines $/ \mathrm{mm}$ and 1800 lines $/ \mathrm{mm}$ grating, and a backilluminated CCD. A 100X objective with respective numerical aperture of 0.9 and 1.4 , and a $50-\mu \mathrm{m}$ core diameter multimode fiber acting as a pinhole, were used to collect the signal. The expected lateral spatial resolution in the $x-y$ plane can reach $150 \mathrm{~nm}$ (num app=1.4) and $250 \mathrm{~nm}$ (num app=0.9). A particular attention was paid to the incident laser power density to prevent local heating effects that may induce a shift of the Raman peaks. UPS measurements for the study of the work function have been performed using a PHI 5000 Versaprobe spectrometer (Physical Electronics) operating at a base pressure of $10^{-9} \mathrm{mbar}$, using a He I discharge lamp $(\mathrm{h} v=21.2 \mathrm{eV})$.

\section{Results and discussion}

The typical morphology of the graphene sample is displayed in the AFM images of Figure 1. The step direction and terrace width were determined by the incidental misorientation of the substrate surface with respect to the crystallographic (0001) plane. The surface is highly uniform over a large scale with atomically flat, $3 \mu \mathrm{m}$ large terraces and $1.8 \mathrm{~nm}$ high steps. On defect-free areas of the sample, the terraces typically extend undisturbed over $50 \mu \mathrm{m}$ in length. In AFM phase mode (Figure1 (b)), the homogenous contrast indicates the thickness homogeneity, that corresponds to a graphene monolayer.

Figure 2 displays the typical spectra over the range $1200-3000 \mathrm{~cm}^{-1}$ of the pristine graphene and NA doped graphene samples ${ }^{23}$. For each sample, to reduce the possible errors affecting the peaks positions and the intensity due to local inhomogeneity, we collected the average Raman spectra over an area of $1 \mu \mathrm{m}^{2}$, before (pristine) and after the doping. We observed the main features of graphene as well as the second-order Raman signal that originates from the $\mathrm{SiC}$ substrate visible between 1200 and $3000 \mathrm{~cm}^{-1}$. Graphene contributes to Raman spectra with three main peaks: i) D band at $\sim 1350 \mathrm{~cm}^{-1}$ (defect induced mode), ii) G band at $\sim 1590 \mathrm{~cm}^{-1}$ (in-plane vibrational mode) and, iii) the $2 \mathrm{D}$ band (two-phonon mode) at $\sim 2700 \mathrm{~cm}^{-124,25}$. The pristine sample does not present any noticeable $\mathrm{D}$ band indicating a low density of defects and a high crystalline phase i.e. $\mathrm{I}_{\mathrm{D}} / \mathrm{I}_{\mathrm{G}}$ 
ratio $\sim 0.13$. On the other hand, all NA doped samples exhibit a higher intensity $\mathrm{D}$ band. The $\mathrm{I}_{\mathrm{D}} / \mathrm{I}_{\mathrm{G}}$ ratio increases from $\sim 0.13$ for the pristine sample to an average of 2.5 for doped samples confirming the alteration of the structure after NA dip. In addition, we note the appearance of a (G+D) band $\sim 2950 \mathrm{~cm}^{-1}$ caused by the disorderinduced feature in $s p^{2}$ carbon associated with defects. The 2D band full width half-maximum (FWHM) increases from $35 \mathrm{~cm}^{-1}$ for the pristine sample to an average of $42 \mathrm{~cm}^{-1}$ for NA15, NA30 and NA70, and raised to $50 \mathrm{~cm}^{-1}$ for the maximum doping for NA100. These Raman shifts clearly indicate a doping effect induced by the NA dip at various concentrations. The bending of the graphene sheet at the kinks of the terraces induces local physical stress on the carbon atoms of the graphene layer that results in an increase in the chemical reactivity of these atoms, and then we can suppose that the incorporation of dopants occurs at the edges states of the epitaxial graphene ${ }^{26,27}$.

We observe that, for the NA15 sample the position of the G-band before and after doping is within the resolution of the Raman system i.e. $\sim 1 \mathrm{~cm}^{-1}$. However, there is a red shift of $14 \mathrm{~cm}^{-1}$ in the position of the 2Dband assigned to an $n$-type doping. The defect induced D band also increases with respect to the pristine sample as expected for doped samples. For this sample, the D band intensity was not changed, mainly because the spot size of the confocal Raman was smaller than the size of the terraces affected by the edges of epitaxial graphene, thus inducing a D-band intensity even before doping. In the case of NA30 sample, we note a significant red shift for both the G-band and the 2D-band positions, of 8 and $19 \mathrm{~cm}^{-1}$ respectively, showing a strong $n$-type doping behavior ${ }^{28}$. The D-band also shows a prominent intensity increment after doping with NA30, which is due to the $s p^{3}$-activation by nitrogen intercalation in the conjugated $s p^{2}$ network of graphene. Note that the epitaxial graphene is intrinsically $n$-doped due the charge transfer occurring from the SiC substrate through the interface layer ${ }^{29}$. This point will be further discussed in the UPS section. NA70 sample does not present a relevant shift in the G-band position. However, the red shift of the 2D-band is reduced to $6 \mathrm{~cm}^{-1}$, denoting a reduced $n$-type doping effect as opposed to NA15 and NA30. NA100 shows a different behavior as the G-band position remains stable but the $2 \mathrm{D}$ band exhibits a blue shift of $11 \mathrm{~cm}^{-1}$ indicating $p$-type like doping ${ }^{30}$. We believe that this effect at high NA concentrations results from a balance between two different simultaneous effects, i.e. $p$ and $n$ type doping from physisorption of the molecules and nitrogenation reactions near the terraces, respectively.

To evaluate the electronic quality of our graphene layers, we determined the carrier mobility of the pristine and NA100\% doped graphene using van der Pauw measurements at room temperature. The electron density in several areas of these samples was typically $9 \times 10^{12} \mathrm{~cm}^{-2}$ and $6 \times 10^{12} \mathrm{~cm}^{-2}$ respectively, with a macroscopically averaged electron mobility inferred from the Hall voltage estimated at about $2500 \mathrm{~cm}^{2} /(\mathrm{Vs})$ and $1700 \mathrm{~cm}^{2} /(\mathrm{Vs})$ respectively. We have measured in previous works the electronic properties on such a monolayer graphene using the same growth mechanism with hydrogen, nitrogen introduced in-situ and ammonia (liquid or vapor) as doping media ${ }^{18}, 22$. The measurements showed outstanding properties including quantum Hall effect and the high mobility reached up to $11000 \mathrm{~cm}^{-2} \cdot \mathrm{V}^{-1} \cdot \mathrm{s}^{-1}$ at $1.7 \mathrm{~K}$ for carrier density $\sim 10^{12} \mathrm{~cm}^{-2}$. The lowest mobility achieved was $1400 \mathrm{~cm}^{-2} \cdot \mathrm{V}^{-1} \cdot \mathrm{s}^{-1}$ at $300 \mathrm{~K}^{17}$. Note that Günes et al ${ }^{14}$ achieved a sheet resistance of $100 \Omega / \square$ using $\mathrm{HNO}_{3}$ doping on CVD graphene. These data clearly indicate that although doping alters to some extent the lattice of graphene, it does not render the material electronically poor. The orders of magnitude are sufficient for many mainstream applications where contacts are required ${ }^{31}, 32$. 
For that purpose and to complete the picture of the electronic properties of doped monolayer epitaxial graphene, we performed UPS measurements in order to monitor the WF as function of the NA concentration. The UPS spectra around the secondary electron threshold region of the graphene layer treated by NA solutions are shown in Figure 3(a). The secondary electron threshold was determined by extrapolation between the background and straight solid lines in the secondary electron threshold region of the UPS spectra. The work function was determined from the secondary electron threshold using the equation $\Phi=\mathrm{h} v-\mathrm{E}_{\mathrm{th}}$, where $\mathrm{h} v$ and $\mathrm{E}_{\mathrm{th}}$ are the photon energy of excitation light (He I discharge lamp, $21.2 \mathrm{eV}$ ) and the secondary electron threshold energy, respectively. Figure 3(b) shows the work function of graphene pristine and $\mathrm{HNO}_{3}$ doped graphene. Decomposition of $\mathrm{HNO}_{3}$ molecules takes place upon the interaction with the graphene layer where the active edge-state electrons play a catalytic role. While the $\mathrm{HNO}_{3}$ concentration increases, the amount of species physisorbed onto the graphene layer increases. The work function of pristine graphene layer is $4.3 \mathrm{eV}$ as reported by different groups ${ }^{33,34}$. Subsequently, the work function decreases from $4.3 \mathrm{eV}$ to $4.1 \mathrm{eV}$ after doping with $15 \%$ NA. The WF further decreases to $3.8 \mathrm{eV}$ with $30 \%$ NA. Note that this reduction implies an $n$-type doping that is consistent with the variation observed on the Raman spectra on Figure 2, suggesting an increase of the nitrogen content between pristine and 30\% NA. Beyond 30\% NA, the WF increases first to $4.05 \mathrm{eV}$ at $70 \%$ NA and reaches $4.41 \mathrm{eV}$ for pure NA. For the latter we also observed a blue shift on the Raman spectrum of NA100 confirming a $p$-type doping that compensates the initial $n$-type nature of the sample. The changes of the Fermi level position with the NA concentration is illustrated in Figure 3 (b). Our results clearly indicate that for low NA concentration, the nitrogenation reduces the WF towards the $n$-type doping as the nitrogen content (donor) increases in the sample ${ }^{35}$. This is in contrast to the observation of Das et al where the WF presents a monotonic increase as a function of NA\%. Note that the graphene utilized here is initially n-type due to a residual doping from the $\mathrm{SiC}$ substrate. We believe that the first $\mathrm{HNO}_{3}$ molecules dissociate and saturate all available defects sites or steps edges. This induces a nitrogen doped graphene layer (NA15\% and NA30\%) and therefore decreases the $\mathrm{WF}^{35}$. On the other hand, beyond $\mathrm{NA} 30 \%$, the $\mathrm{HNO}_{3}$ can physisorb on the surface inducing a $p$-type doping, lowering the Fermi level and as a result increasing the WF. This was confirmed by $a b$ initio calculations describing the electronic structure of graphene on $\mathrm{SiC}$ as well as the interaction with the $\mathrm{HNO}_{3}$ molecules were performed using a very efficient DFT localized orbital molecular dynamic technique $\left(\right.$ FIREBALL) ${ }^{36}$. Numerical localized orbital basis sets of $\mathrm{sp}^{3}$ for $\mathrm{C}, \mathrm{N}, \mathrm{O}$ and $\mathrm{Si}$, and $\mathrm{s}$ for $\mathrm{H}$ were used with spatial cutoff radii (in atomic units) $\mathrm{s}=4.5, \mathrm{p}=4.5(\mathrm{C}), \mathrm{s}=4.2, \mathrm{p}=4.2(\mathrm{~N}), \mathrm{s}=3.3, \mathrm{p}=3.8(\mathrm{O}), \mathrm{s}=4.8, \mathrm{p}=5.4$ (Si) and $\mathrm{s}=4.1(\mathrm{H})^{37}$. In this study we have considered supercells of $5 \mathrm{ML} \mathrm{SiC}(0001)$, with a zero-layer graphene and a $\mathrm{AB}$ stacked graphene plane on top. The lateral size roughly corresponds to a $4 \mathrm{x} 4$ unit cell of graphene. The bottom layer is saturated with hydrogen atoms. On top of the supercell, we have set from 1 to 4 $\mathrm{HNO}_{3}$ molecules per unit cell to observe the graphene doping evolution with the molecular concentration, as represented in Figures 4 (a), (b) and (c). The geometry of those systems has then been relaxed at $0 \mathrm{~K}$, using a sample of $32 \mathrm{k}$-points in the surface Brillouin zone, as previously used in similar calculations ${ }^{38}$, maintaining the last three bottom layers in bulk positions. The equilibrium configuration is obtained when forces are less than $0.01 \mathrm{eV} / \AA$. The final distances between the molecules and the graphene plane have been determined using the well-known LCAO-S ${ }^{2}+$ vdW formalism ${ }^{39}, 40,41$ which specifically takes into account van der Waals interaction in the frame of DFT. Finally, a set of 300 special $\mathrm{k}$ points along the $\Gamma-\mathrm{K}-\mathrm{M}$ path has been used for the band structure calculations on the optimized geometries. These calculations aim at reproducing the physisorbed 
process leading to a $p$-doping, as we did not model the graphene terraces. Results of the band structure calculations are represented in Figures 4 (d), (e) and (f), corresponding to one, two or four deposited $\mathrm{HNO}_{3}$ molecules. We can observe a variation of the graphene doping from $-350 \mathrm{meV}$ for one molecule (which represents already a $p$-type doping with respect to the pristine situation at $-400 \mathrm{meV}$ ), to $-180 \mathrm{meV}$ for two and until $+190 \mathrm{meV}$ for four molecules. Consequently, the calculations also confirm a $p$-type doping for the physisorption process in good agreement with the WF variation recorded experimentally.

\section{Conclusions}

We have investigated the doping strength of nitric acid on monolayer graphene grown on SiC (0001). The Raman measurements confirm the doping induced by various concentration of nitric acid and indicate the incorporation of atoms into the graphene lattice. The UPS measurements showed that $n$-type and $p$-type doping are competitive processes and the work function of graphene layer can be continuously tuned from $4.3 \mathrm{eV}$ to 4.4 $\mathrm{eV}$ with a minimum of $3.8 \mathrm{eV}$ upon depositing the molecular acceptor $\mathrm{HNO}_{3}$. This observation is supported by DFT calculations considering the physisorption of $\mathrm{HNO}_{3}$ molecules. This simple approach allows the modulation of WF and could permit the design of tuneable electrodes to improve the carrier injection as well as band gap-engineered heterostructures.

This work is supported by a public grant overseen by the French National Research Agency (ANR) as part of the "Investissements d'Avenir" program (Labex NanoSaclay, reference: ANR-10-LABX-0035), as well as the the French Ministère des affaires étrangères et européennes (MAEE), the Centre National de la Recherche Scientifique (CNRS) through the STIC ASIA programme grant 3226/DGM/ATT/RECH. The XPS/UPS equipment was supported by a grant from Region Ile-de-France.

\section{Figures captions:}

Figure 1: (a) and (b) AFM topography and phase of pristine graphene sample.

Figure 2: Raman spectra of pristine, NA15, NA30, NA70 and NA100 samples. D peak magnitude appears with NA concentration indicating the creation of defects due to nitrogen incorporation. $(\mathrm{G}+\mathrm{D})$ band indicate a similar effect. Raman shifts indicated by arrows evidence the doping trends. The presence of the G and 2D bands across the overall area indicates homogenous growth of graphene.

Figure 3: (a) UPS data for pristine, NA30, NA70 and NA100 samples indicating the WF shift as a function of nitric acid concentration. (b) Illustration of the Fermi level shift in the graphene Dirac cones. 
Figure 4: (a), (b), and (c) atomic structures used for the calculations, considering one graphene layer on top of a $\mathrm{SiC}(0001)$ surface with graphene buffer layer, and one, two and four $\mathrm{HNO}_{3}$ molecules respectively, (d), (e) and (f), corresponding band structures showing the evolution of the graphene doping from $n$ to $p$-type. The graphene Dirac cone is highlighted in red for the three configurations. 

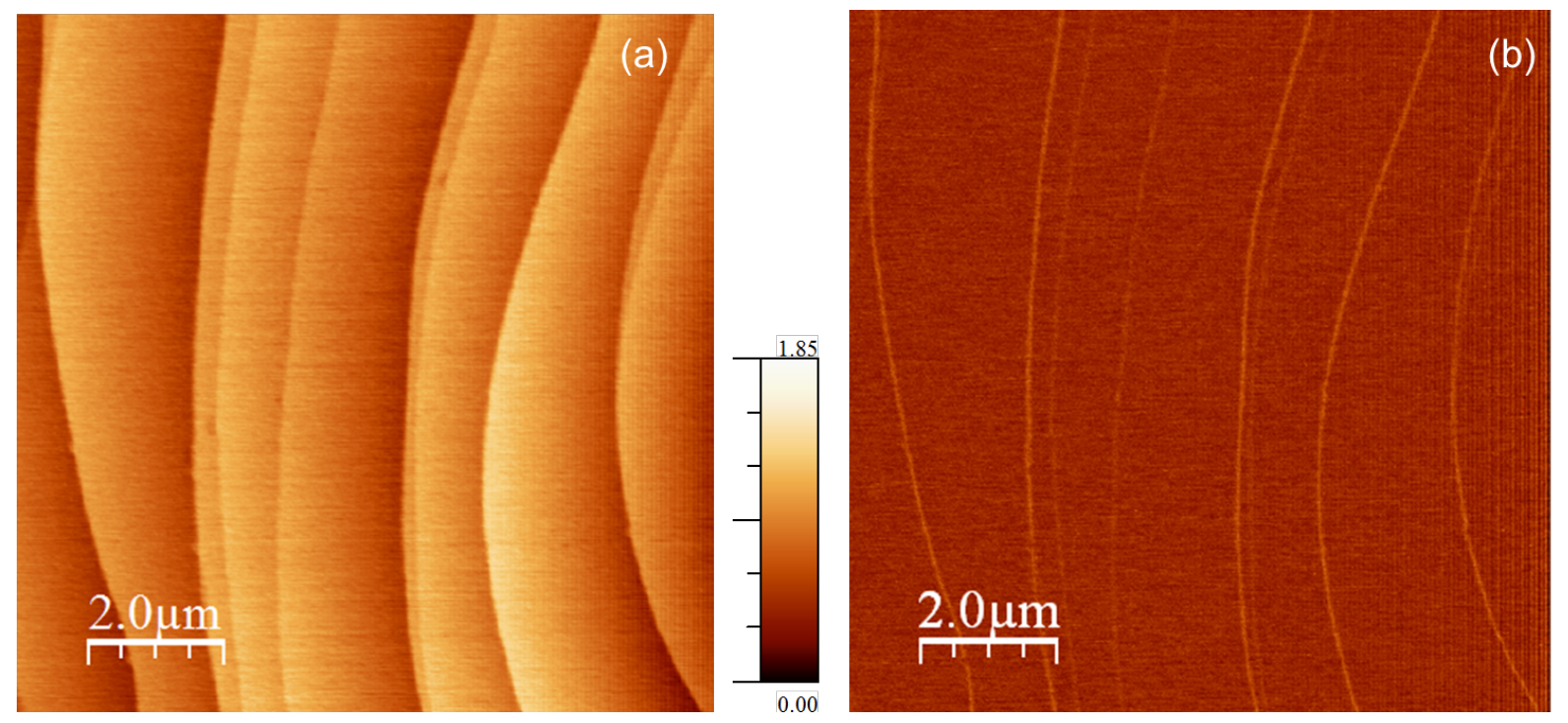

Figure 1

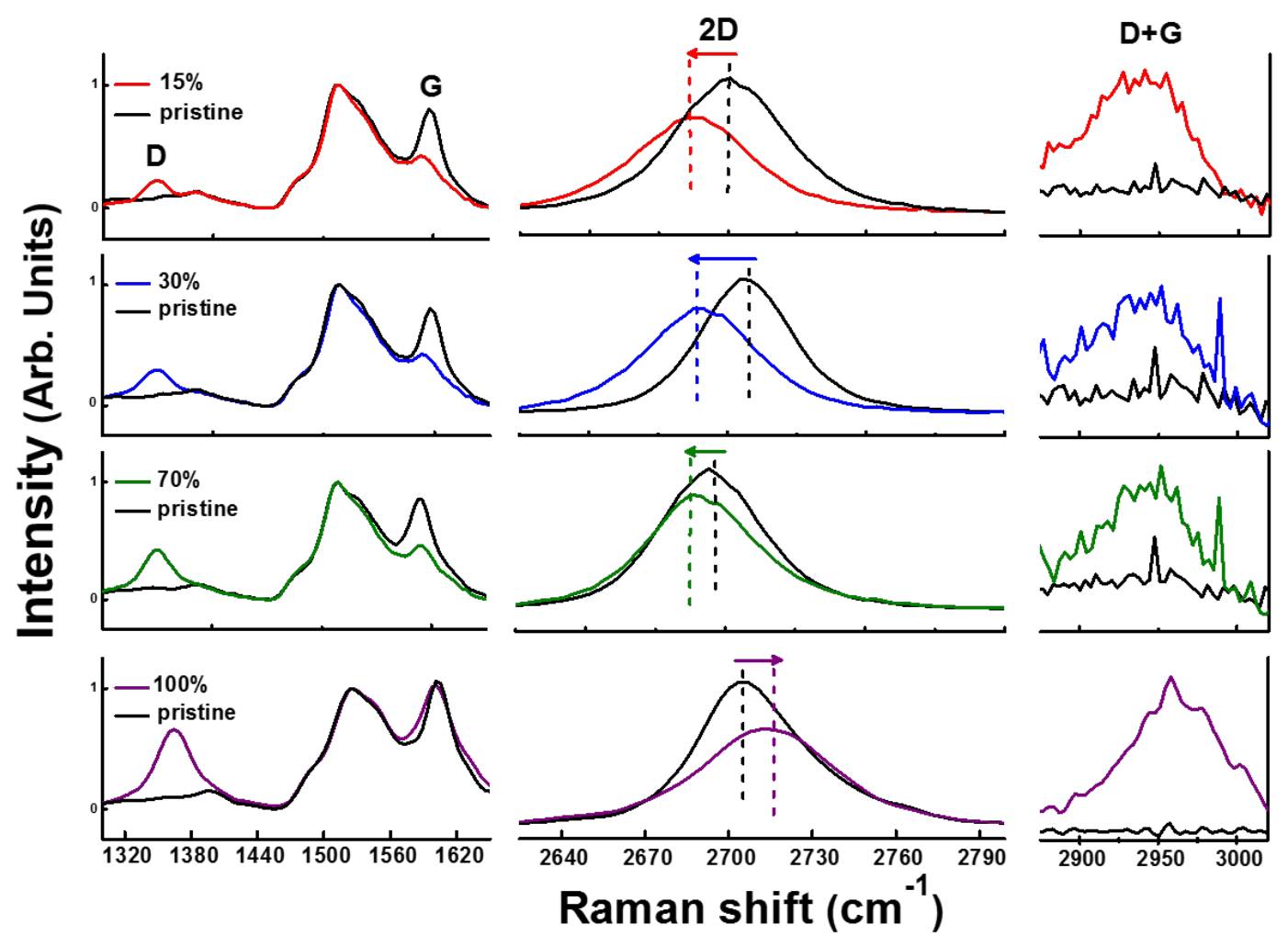

Figure 2 


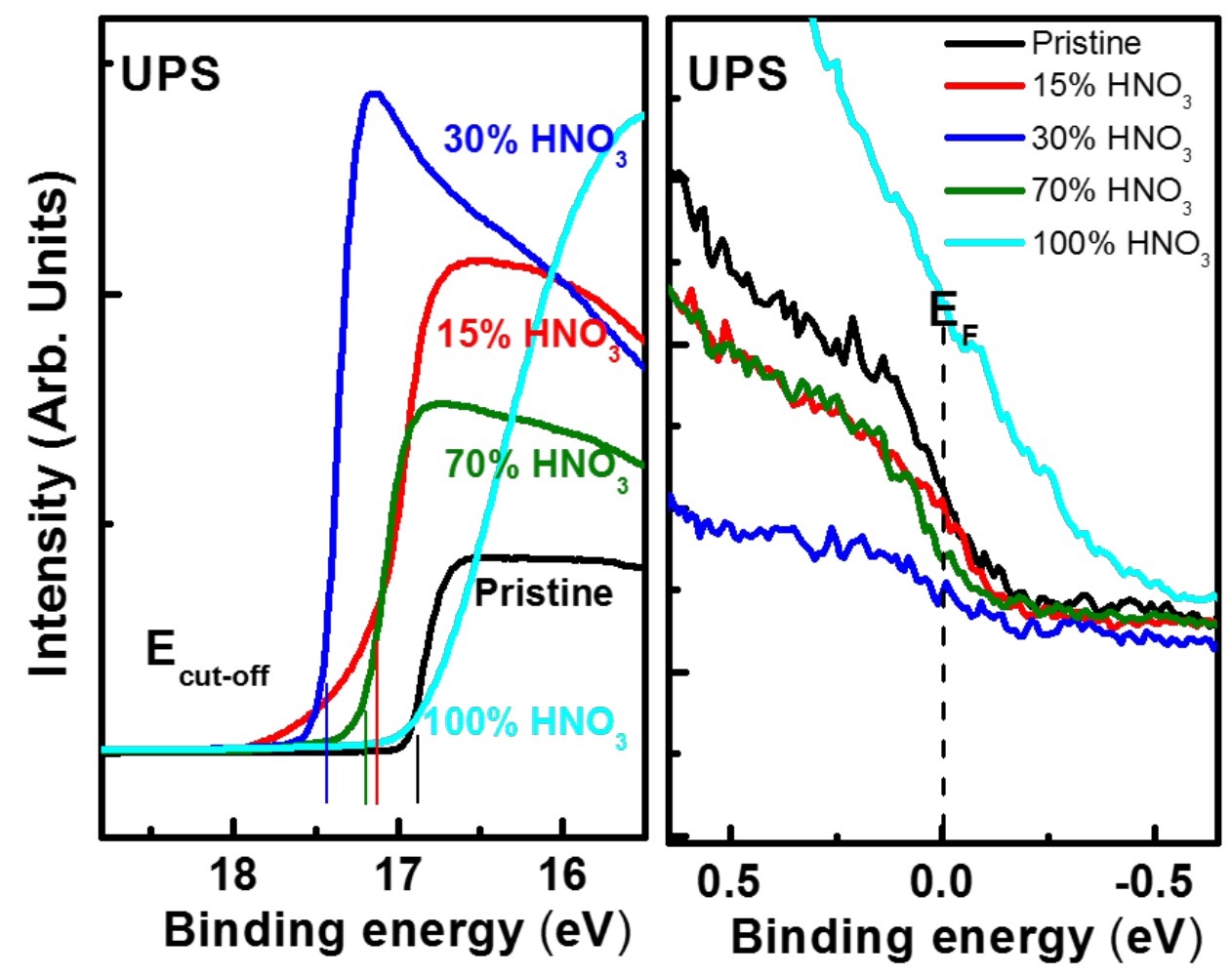

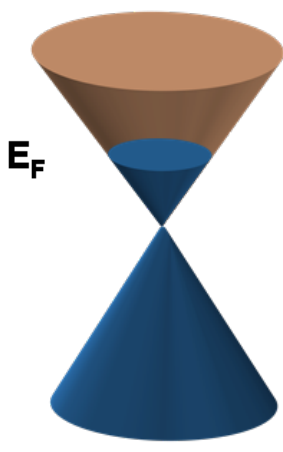

$W F=4.3 e V$

Pristine

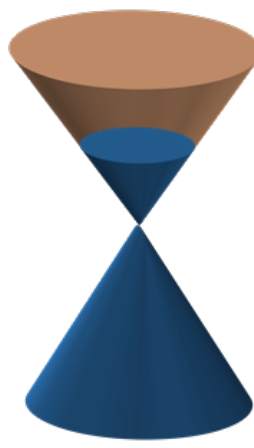

$W F=4.05 \mathrm{eV}$ NA15

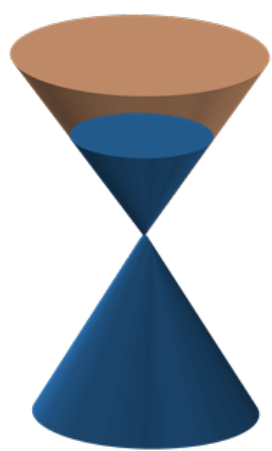

$W F=3.8 \mathrm{eV}$

NA30

Figure 3

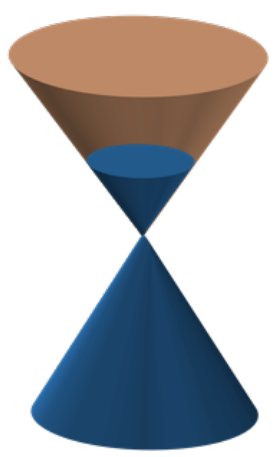

$\mathrm{WF}=4 \mathrm{eV}$ NA70

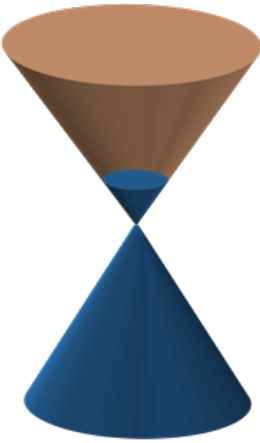

$W F=4.41 \mathrm{eV}$ NA100 
(a)

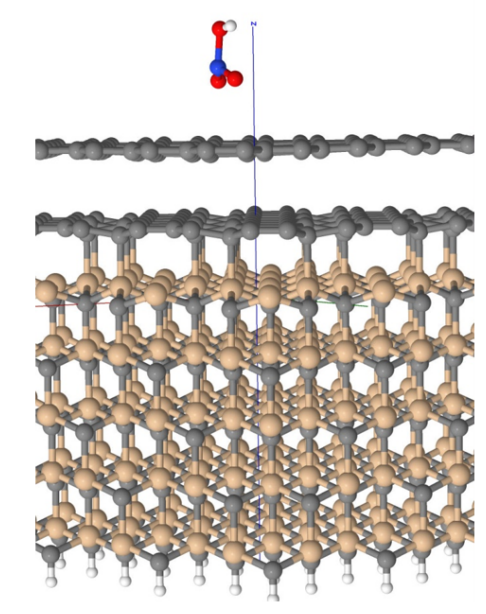

(d)

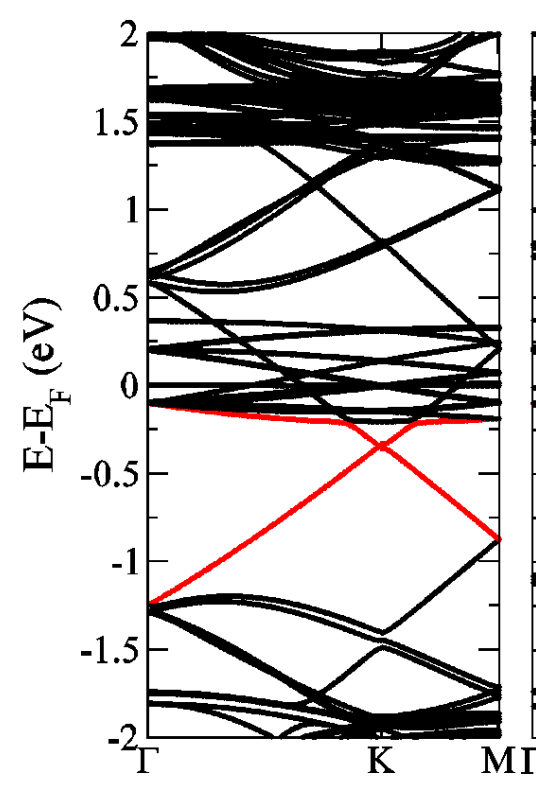

(b)

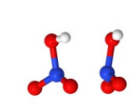

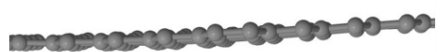

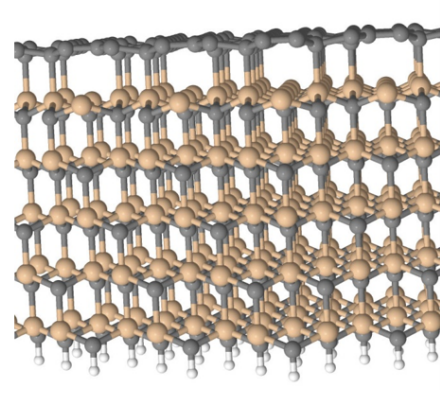

(c)

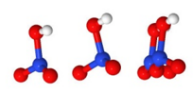

$-05-3-00-00=00-0000000$

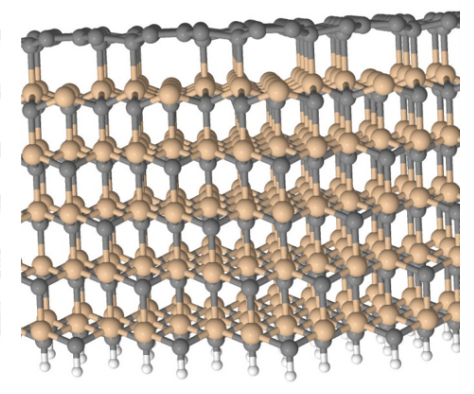

(e)

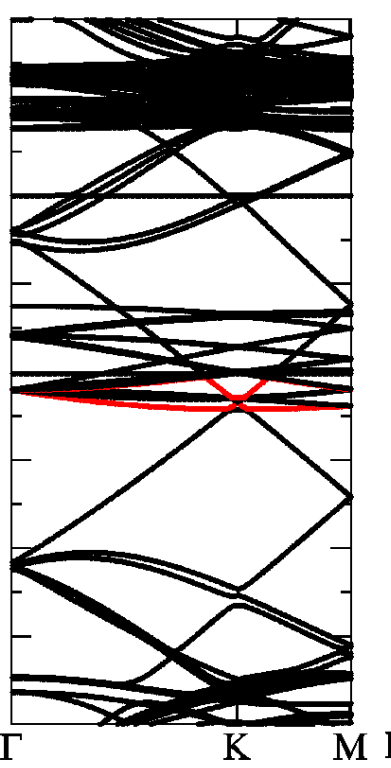

(f)

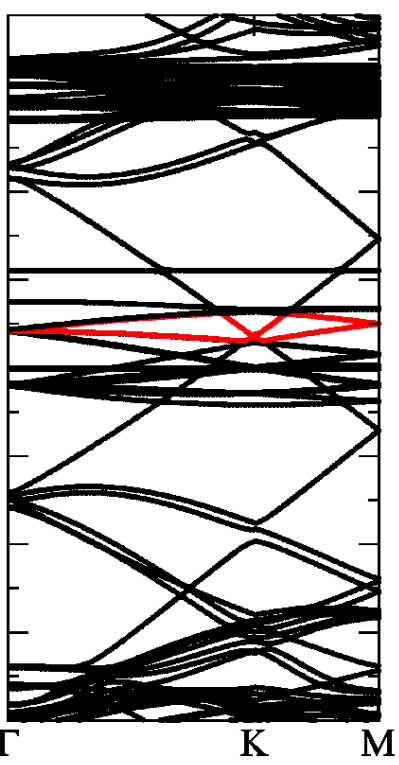

Figure 4 


\section{REFERENCES}

${ }^{1}$ K.S. Novoselov, A.K. Geim, S.V. Morozov, D. Jiang, M.I. Katsnelson, I.V. Grigorieva, S.V. Dubonos, and A.A. Firsov, Nature 438, 197 (2005).

${ }^{2}$ Y. Zhang, Y.-W. Tan, H.L. Stormer, and P. Kim, Nature 438, 201 (2005).

${ }^{3}$ Y. Zhang, T.-T. Tang, C. Girit, Z. Hao, M.C. Martin, A. Zettl, M.F. Crommie, Y.R. Shen, and F. Wang, Nature 459, 820 (2009).

${ }^{4}$ G. Eda, G. Fanchini, and M. Chhowalla, Nat. Nanotechnol. 3, 270 (2008).

${ }^{5}$ J. Guignard, D. Leprat, D.C. Glattli, F. Schopfer, and W. Poirier, Phys. Rev. B 85, (2012).

${ }^{6}$ E. Pallecchi, M. Ridene, D. Kazazis, F. Lafont, F. Schopfer, W. Poirier, M.O. Goerbig, D. Mailly, and A. Ouerghi, Sci. Rep. 3, 1791 (2013).

${ }^{7}$ S. Bae, H. Kim, Y. Lee, X. Xu, J.-S. Park, Y. Zheng, J. Balakrishnan, T. Lei, H. Ri Kim, Y.I. Song, Y.-J. Kim, K.S. Kim, B. Özyilmaz, J.-H. Ahn, B.H. Hong, and S. Iijima, Nat. Nanotechnol. 5, 574 (2010).

${ }^{8}$ L.G. De Arco, Y. Zhang, and C. Zhou, Graphene-Synth. Charact. Prop. Appl. (2011).

${ }^{9}$ R. Balog, B. Jørgensen, L. Nilsson, M. Andersen, E. Rienks, M. Bianchi, M. Fanetti, E. Lægsgaard, A. Baraldi, S. Lizzit, Z. Sljivancanin, F. Besenbacher, B. Hammer, T.G. Pedersen, P. Hofmann, and L. Hornekær, Nat. Mater. 9, 315 (2010).

${ }^{10}$ M. Han, B. Özyilmaz, Y. Zhang, and P. Kim, Phys. Rev. Lett. 98, (2007).

${ }^{11}$ M. Liu, X. Yin, E. Ulin-Avila, B. Geng, T. Zentgraf, L. Ju, F. Wang, and X. Zhang, Nature 474, 64 (2011).

${ }^{12}$ F. Güneş, G.H. Han, H.-J. Shin, S.Y. Lee, M. Jin, D.L. Duong, S.J. Chae, E.S. Kim, F. Yao, A. Benayad, J.-Y. Choi, and Y.H. Lee, Nano 06, 409 (2011).

${ }^{13}$ H.-J. Shin, W.M. Choi, D. Choi, G.H. Han, S.-M. Yoon, H.-K. Park, S.-W. Kim, Y.W. Jin, S.Y. Lee, J.M. Kim, J.-Y. Choi, and Y.H. Lee, J. Am. Chem. Soc. 132, 15603 (2010).

${ }^{14}$ F. Günes, H.-J. Shin, C. Biswas, G.H. Han, E.S. Kim, S.J. Chae, J.-Y. Choi, and Y.H. Lee, ACS Nano 4, 4595 (2010).

${ }^{15}$ M.W. Iqbal, A.K. Singh, M.Z. Iqbal, and J. Eom, J. Phys. Condens. Matter 24, 335301 (2012).

${ }^{16}$ M. Na and S.-W. Rhee, Org. Electron. 7, 205 (2006).

${ }^{17}$ E. Pallecchi, F. Lafont, V. Cavaliere, F. Schopfer, D. Mailly, W. Poirier, and A. Ouerghi, Sci. Rep. 4, 4558 (2014).

${ }^{18}$ M. Boutchich, H. Arezki, D. Alamarguy, K.-I. Ho, H. Sediri, F. Güneş, J. Alvarez, J.P. Kleider, C.S. Lai, and A. Ouerghi, Appl. Phys. Lett. 105, 233111 (2014).

${ }^{19}$ S. Das, P. Sudhagar, E. Ito, D. Lee, S. Nagarajan, S.Y. Lee, Y.S. Kang, and W. Choi, J. Mater. Chem. 22, 20490 (2012).

${ }^{20}$ B. Guo, L. Fang, B. Zhang, and J.R. Gong, Insciences J. 80 (2011).

${ }^{21}$ B. Lalmi, J.C. Girard, E. Pallecchi, M. Silly, C. David, S. Latil, F. Sirotti, and A. Ouerghi, Sci. Rep. 4, 4066 (2014).

${ }^{22}$ E. Velez-Fort, C. Mathieu, E. Pallecchi, M. Pigneur, M.G. Silly, R. Belkhou, M. Marangolo, A. Shukla, F. Sirotti, and A. Ouerghi, ACS Nano 6, 10893 (2012).

${ }^{23}$ A. Ouerghi, A. Balan, C. Castelli, M. Picher, R. Belkhou, M. Eddrief, M.G. Silly, M. Marangolo, A. Shukla, and F. Sirotti, Appl. Phys. Lett. 101, 021603 (2012).

${ }^{24}$ A.C. Ferrari, Solid State Commun. 143, 47 (2007).

${ }^{25}$ L.M. Malard, M.A. Pimenta, G. Dresselhaus, and M.S. Dresselhaus, Phys. Rep. 473, 51 (2009).

${ }^{26}$ A. Eckmann, A. Felten, A. Mishchenko, L. Britnell, R. Krupke, K.S. Novoselov, and C. Casiraghi, Nano Lett. 12, 3925 (2012).

${ }^{27}$ F. Banhart, J. Kotakoski, and A.V. Krasheninnikov, ACS Nano 5, 26 (2011).

${ }^{28}$ B. Guo, Q. Liu, E. Chen, H. Zhu, L. Fang, and J.R. Gong, Nano Lett. 10, 4975 (2010).

${ }^{29}$ C. Coletti, C. Riedl, D.S. Lee, B. Krauss, L. Patthey, K. Von Klitzing, J.H. Smet, and U. Starke, Phys. Rev. B 81, 235401 (2010).

${ }^{30}$ C. Casiraghi, Phys. Status Solidi RRL - Rapid Res. Lett. 3, 175 (2009).

${ }^{31}$ P.K. Mohseni, A. Behnam, J.D. Wood, X. Zhao, K.J. Yu, N.C. Wang, A. Rockett, J.A. Rogers, J.W. Lyding, E. Pop, and X. Li, Adv. Mater. 26, 3755 (2014).

32 J. Meyer, P.R. Kidambi, B.C. Bayer, C. Weijtens, A. Kuhn, A. Centeno, A. Pesquera, A. Zurutuza, J. Robertson, and S. Hofmann, Sci. Rep. 4, (2014).

${ }^{33}$ S.-M. Park, S.-N. Yang, K.-J. Kim, K.-H. No, and H.-I. Lee, Bull. Korean Chem. Soc. 31, 2809 (2010).

${ }^{34}$ H. Hibino, H. Kageshima, M. Kotsugi, F. Maeda, F.-Z. Guo, and Y. Watanabe, Phys. Rev. B 79, (2009). 
${ }^{35}$ Z. Luo, S. Lim, Z. Tian, J. Shang, L. Lai, B. MacDonald, C. Fu, Z. Shen, T. Yu, and J. Lin, J. Mater. Chem. 21, 8038 (2011).

${ }^{36}$ J.D. Bozek, G.M. Bancroft, J.N. Cutler, and K.H. Tan, Phys. Rev. Lett. 65, 2757 (1990).

37 J.P. Lewis, P. Jelínek, J. Ortega, A.A. Demkov, D.G. Trabada, B. Haycock, H. Wang, G. Adams, J.K. Tomfohr, E. Abad, H. Wang, and D.A. Drabold, Phys. Status Solidi B 248, 1989 (2011).

${ }^{38}$ M.A. Basanta, Y.J. Dappe, P. Jelínek, and J. Ortega, Comput. Mater. Sci. 39, 759 (2007).

${ }^{39}$ D. Pierucci, H. Sediri, M. Hajlaoui, E. Velez-Fort, Y.J. Dappe, M.G. Silly, R. Belkhou, A. Shukla, F. Sirotti, N. Gogneau, and A. Ouerghi, Nano Res. 8, 1026 (2015).

${ }^{40}$ Y.J. Dappe, J. Ortega, and F. Flores, Phys. Rev. B 79, (2009).

${ }^{41}$ A.J. Martínez-Galera, N. Nicoara, J.I. Martínez, Y.J. Dappe, J. Ortega, and J.M. Gómez-Rodríguez, J. Phys. Chem. C 118, 12782 (2014). 